PROCEEDINGS OF THE

AMERICAN MATHEMATICAL SOCIETY

Volume 135, Number 12, December 2007, Pages 3933-3941

S 0002-9939(07)08978-2

Article electronically published on August 2, 2007

\title{
GLOBAL EXISTENCE OF SOLUTIONS TO SHIGESADA-KAWASAKI-TERAMOTO CROSS-DIFFUSION SYSTEMS ON DOMAINS OF ARBITRARY DIMENSIONS
}

\author{
PHAN VĂN TUỘC
}

(Communicated by David S. Tartakoff)

\begin{abstract}
We consider a strongly coupled nonlinear parabolic system which arises in population dynamics in $n$-dimensional domains $(n \geq 1)$. Global existence of classical solutions under certain restrictions on the coefficients is established.
\end{abstract}

\section{INTRODUCTION}

In this paper, we study the following cross-diffusion system in population dynamics:

$$
\left\{\begin{aligned}
u_{t} & =\Delta\left[\left(d_{1}+\alpha v+\gamma u\right) u\right]+u\left(a_{1}-b_{1} u-c_{1} v\right) & & \text { in } \Omega \times[0, \infty), \\
v_{t} & =\Delta\left[\left(d_{2}+\delta v\right) v\right]+v\left(a_{2}-b_{2} u-c_{2} v\right) & & \text { in } \Omega \times[0, \infty), \\
\frac{\partial u}{\partial \nu} & =\frac{\partial v}{\partial \nu}=0 & & \text { in } \partial \Omega \times[0, \infty), \\
u(x, 0) & =u_{0}(x) \geq 0, \quad v(x, 0)=v_{0}(x) \geq 0 & & \text { in } \Omega,
\end{aligned}\right.
$$

where $\Omega$ is an open bounded domain in $\mathbb{R}^{n}$ (for $n \in \mathbb{N}$ ) with smooth boundary $\partial \Omega$, $\Delta=\sum_{i=1}^{n} \frac{\partial^{2}}{\partial x_{i}{ }^{2}}$ is the Laplacian, $\partial / \partial \nu$ denotes the directional derivative along the outward normal on $\partial \Omega$ and $\alpha, \gamma, \delta, a_{i}, b_{i}, c_{i}$ and $d_{i}(i=1,2)$ are given positive constants. The system (1.1) is a special case of models proposed by Shigesada, Kawasaki and Teramoto [15] in 1979 when they studied the segregation phenomena of competing species. In this system, $u$ and $v$ are nonnegative functions which represent population densities of two competing species, $d_{1}$ and $d_{2}$ are respectively their diffusion rates, $a_{1}$ and $a_{2}$ denote intrinsic growth rates, $b_{1}$ and $c_{2}$ account for intraspecific competitions, and $b_{2}$ and $c_{1}$ are inter-specific competition coefficients. The homogeneous Neumann boundary condition means there is no migration crossing the boundary $\partial \Omega$. When $\alpha=\delta=\gamma=0$, (1.1) reduces to the well-known LotkaVolterra competition-diffusion system. The parameters $\gamma$ and $\delta$ are self-diffusion rates, and $\alpha$ is a cross-diffusion rate. For more details on the background of this model, see reference [15].

Local existence (in time) of solutions to (1.1) was established by Amann in a series of important papers [1, [2, [3. His result can be summarized as follows.

Received by the editors April 12, 2006 and, in revised form, October 8, 2006. 2000 Mathematics Subject Classification. Primary 35B50, 35K50, 35K55, 35K57.

Key words and phrases. Maximum principles, cross-diffusion systems, global existence. 
Theorem 1.1. Assume $p>n, u_{0}, v_{0} \in W_{p}^{1}(\Omega)$. The system (1.1) has a unique nonnegative smooth solution $u, v \in C\left([0, T), W^{1, p}(\Omega)\right) \cap C^{\infty}\left((0, T), C^{\infty}(\bar{\Omega})\right)$ with maximal existence time $T$. Moreover, if the solution $(u, v)$ satisfies the estimate

$$
\sup _{0 \leq t<T}\|u(., t)\|_{W_{p}^{1}(\Omega)}<\infty \text { and } \sup _{0 \leq t<T}\|v(., t)\|_{W_{p}^{1}(\Omega)}<\infty,
$$

then $T=\infty$.

Global existence (in time) of (1.1) has recently received great attention (see [5], [6], 11] and [13). When $n=2, \mathrm{Lou}, \mathrm{Ni}$ and Wu proved in 1998 that the system (1.1) has a unique global classical solution (see [13]). In 2003, Choi, Lui and Yamada considered the problem for $\delta=0$ and any $n$. However, they only obtained the global existence of the solution to (1.1) when the coefficient $\alpha$ is sufficiently small (see [5]). Then in 2004, Choi, Lui and Yamada continued their work and they showed that the solution to (1.1) exists globally for either $\delta=0$ or $\delta>0$ and $n<6$ (see [6]). In these papers, to get the boundedness of the solution of (1.1), which is crucial in proving (1.2), the authors first obtained $L^{p}$-estimates of the solution and then used the Sobolev embeddings and standard regularity results of parabolic equations. Therefore, they have a restriction on the dimension $n$ of $\Omega$.

In a different approach, the system (1.1) can be written in the following form:

$$
\begin{cases}u_{t}=\nabla \cdot[P(u, v) \nabla u+R(u) \nabla v] & +u\left(a_{1}-b_{1} u-c_{1} v\right), \\ v_{t}=\nabla \cdot[Q(v) \nabla v] & +v\left(a_{2}-b_{2} u-c_{2} v\right),\end{cases}
$$

where

$$
P(u, v)=d_{1}+\alpha v+2 \gamma u, \quad Q(v)=d_{2}+2 \delta v, \quad R(u)=\alpha u .
$$

Using test function techniques, Le and Nguyen [11 obtained some global existence results with arbitrary $n$ for (1.3). Their method is constructive. However, their results (see Theorem 1.1 of [11]) need more various restrictions on the coefficients than our results here (Theorem 1.2 below).

For the system (1.1), it is easy to see that maximum principles can be applied to the second equation of (1.1) to get the boundedness of $v$ (see Lemma 2.1 below). However, to get the control of solution $u$, maximum principles are not available for this system. This is the main difficulty in studying the global existence of (1.1) (see Section 10 of [4). In this paper, contrary to the result of Choi, Lui and Yamada [6], which has a restriction on $n$ when $\delta>0$, we show that, if the self-diffusion coefficient $\delta>0$ is sufficiently large, it helps control the solution $u$ of (1.1). Our idea is to introduce a new function that relates the two equations of (1.1), and this function allows us to use maximum principles to get the boundedness of the solution $u$ of (1.1) (see (2.9) and Theorem 2.1 below). Our approach only makes use of maximum principles and does not need $L^{p}$-estimates of the solution; it is therefore elementary and independent of $n$. Moreover, our method can be generalized to more general abstract equations. We summarize our results in the following theorem.

Theorem 1.2. Suppose that $u_{0} \geq 0, v_{0} \geq 0$ satisfy zero Neumann boundary conditions and belong to $C^{2+\lambda}(\bar{\Omega})$ for some $\lambda>0$. Assume either (i) $\alpha<2 \delta$ or (ii) $\alpha=2 \delta$ and $d_{1} \leq d_{2}$. Then (1.1) possesses a unique nonnegative solution $u, v \in C^{2+\lambda,(2+\lambda) / 2}(\bar{\Omega} \times[0, \infty))$.

The paper is organized as follows. The boundedness of the solution $(u, v)$ of (1.1) obtained by maximum principles will be presented in Section 2. In Section 3, we give a proof of Theorem 1.2 . 


\section{Preliminary Results}

Throughout this paper, $(u, v)$ denotes the nonnegative local smooth solution to (1.1), whose existence is assured by Amann's Theorem (Theorem 1.1), and $T$ denotes its maximal existence time and $Q_{T}=\Omega \times[0, T)$. Also, standard notation for function spaces is used. In particular, $W_{p}^{1}(\Omega)(p>1)$ is a standard Sobolev space and $W_{p}^{2,1}\left(Q_{T}\right)$ is a space consisting of measurable functions $\varphi$ on $Q_{T}$ such that $\varphi, \varphi_{t}, \varphi_{x_{i}}, \varphi_{x_{i} x_{j}}$ are in $L^{p}\left(Q_{T}\right)$ for $i, j=1,2, \ldots, n$ with norm

$$
\|\varphi\|_{W_{p}^{2,1}\left(Q_{T}\right)}=\|\varphi\|_{L^{p}\left(Q_{T}\right)}+\left\|\varphi_{t}\right\|_{L^{p}\left(Q_{T}\right)}+\sum_{i=1}^{n}\left\|\varphi_{x_{i}}\right\|_{L^{p}\left(Q_{T}\right)}+\sum_{i, j=1}^{n}\left\|\varphi_{x_{i} x_{j}}\right\|_{L^{p}\left(Q_{T}\right)} .
$$

First, applying maximum principles (see [8] or [14) to (1.1) we have the following lemma (see also Lemma 2.1 of [13] or Lemma 2.1 of [6] for more details).

Lemma 2.1. There exists a positive constant $m$ such that

$$
0 \leq u \text { and } 0 \leq v \leq m \text { in } Q_{T} .
$$

Now, we shall write the system (1.1) in a matrix form. For simplicity, we denote

$$
\begin{aligned}
& \phi_{1}(u, v)=u\left(a_{1}-b_{1} u-c_{1} v\right), \\
& \phi_{2}(u, v)=v\left(a_{2}-b_{2} u-c_{2} v\right),
\end{aligned}
$$

and

$$
M(\nabla u, \nabla v)=\left(\begin{array}{ll}
2 \gamma \nabla u & 2 \alpha \nabla u \\
0 & 2 \delta \nabla v
\end{array}\right), \quad D(u, v)=\left(\begin{array}{ll}
P(u, v) & R(u) \\
0 & Q(v)
\end{array}\right)
$$

where

$$
P(u, v)=d_{1}+\alpha v+2 \gamma u, \quad Q(v)=d_{2}+2 \delta v, \quad R(u)=\alpha u .
$$

Then the system (1.1) becomes

$$
\left(\begin{array}{c}
u \\
v
\end{array}\right)_{t}=D(u, v)\left(\begin{array}{c}
\Delta u \\
\Delta v
\end{array}\right)+M(\nabla u, \nabla v)\left(\begin{array}{c}
\nabla u \\
\nabla v
\end{array}\right)+\left(\begin{array}{c}
\phi_{1}(u, v) \\
\phi_{2}(u, v)
\end{array}\right) .
$$

Let

$$
f(u, v)=\frac{P-Q}{R}=\frac{d+a u-b v}{u}, \quad d=\frac{d_{1}-d_{2}}{\alpha}, \quad a=\frac{2 \gamma}{\alpha}, \quad b=\frac{2 \delta-\alpha}{\alpha} .
$$

Then, we see that the vector $\langle f, 1\rangle$ is a left eigenvector of $D$ with eigenvalue $P(u, v)$, i.e.,

$$
\langle f, 1\rangle \cdot D(u, v)=P(u, v) \cdot\langle f, 1\rangle, \quad \forall(u, v) .
$$

We also denote $\Gamma=\left\{(u, v) \in \mathbb{R}^{2}: 0 \leq v \leq m, 0 \leq u\right\}$ and $\Gamma_{K}=\left\{(u, v) \in \mathbb{R}^{2}: 0 \leq\right.$ $v \leq m, K<u\}$ for any positive number $K$. Because $a>0$ and $a b^{2}>0$ if $b \neq 0$, we can choose $K_{0}>0$ sufficiently large so that

$$
d+a u-b v>0 \text { and } b[d(b-1)+a b u-b(b-1) v]>1 \text { if } b \neq 0
$$

for $0 \leq v \leq m$ and $u \geq K_{0} / 2$. Now, let $G(u, v)=\psi(u) g(u, v)$ for $(u, v) \in \Gamma$, where

$$
g(u, v)= \begin{cases}(b-1) \log \frac{u^{b}}{b[d(b-1)+a b u-b(b-1) v]} & \text { if } b \neq 0 \text { and } b \neq 1 \\ \frac{v-d}{u}+a \log u & \text { if } b=1, \\ v+d \log u+a u & \text { if } b=0,\end{cases}
$$


and $\psi$ is a smooth function on $\mathbb{R}, 0 \leq \psi \leq 1, \psi(u)=1$ for $u \geq K_{0}$ and $\psi(u)=0$ when $u \leq K_{0} / 2$.

Remark 2.1. When $b \neq 0,1$, the same function as $g(u, v)$ is also used in [1].

Now, we introduce the following lemma.

Lemma 2.2. $G$ is smooth on $\Gamma$. Moreover, on $\Gamma_{K_{0}},\left\langle G_{u}, G_{v}\right\rangle$ is a left eigenvector of $D(u, v)$ with eigenvalue $P(u, v)$,

$$
G_{u}(u, v)=f(u, v) G_{v}(u, v)>0 \text { and } \lim _{u \rightarrow \infty} G(u, v)=\infty .
$$

Proof. By definition of $G$, on $\Gamma_{K_{0}}$ we have

$$
G_{v}(u, v)= \begin{cases}\frac{b^{2}(b-1)^{2}}{b[d(b-1)+a b u-b(b-1) v]} & \text { if } b \neq 0 \text { and } b \neq 1, \\ \frac{1}{u} & \text { if } b=1, \\ 1 & \text { if } b=0,\end{cases}
$$

and $G_{u}(u, v)=f(u, v) G_{v}(u, v)$. Therefore, the lemma follows directly from (2.4), (2.5) and the construction of $G$.

Now we introduce

$$
w(x, t)=G(u(x, t), v(x, t))
$$

We have

$$
\Delta w=G_{u} \Delta u+G_{v} \Delta v+G_{u u}|\nabla u|^{2}+G_{v v}|\nabla v|^{2}+2 G_{u v} \nabla u \nabla v .
$$

By (2.2), (2.4), Lemma 2.2 and (2.10) we see that for any $(x, t) \in Q_{T}$ such that $u(x, t)>K_{0}$,

$$
\begin{aligned}
w_{t}(x, t)= & G_{u} u_{t}+G_{v} v_{t}=\left\langle G_{u}, G_{v}\right\rangle \cdot\left(\begin{array}{c}
u \\
v
\end{array}\right)_{t} \\
=\left(G_{u} \Delta u+\right. & \left.G_{v} \Delta v\right) P+2 \gamma G_{u}|\nabla u|^{2}+2 \alpha G_{u} \nabla u \nabla v \\
& \quad+2 \delta G_{v}|\nabla v|^{2}+G_{u} \phi_{1}+G_{v} \phi_{2} \\
= & P \Delta w+\left(2 \gamma G_{u}-P G_{u u}\right)|\nabla u|^{2}+2\left(\alpha G_{u}-P G_{u v}\right) \nabla u \nabla v \\
& +\left(2 \delta G_{v}-P G_{v v}\right)|\nabla v|^{2}+G_{u} \phi_{1}+G_{v} \phi_{2} .
\end{aligned}
$$

For any $(x, t) \in Q_{T}$, we write

$$
\begin{gathered}
h(x, t)=\left[\left(2 \gamma G_{u}-P G_{u u}\right)|\nabla u|^{2}+2\left(\alpha G_{u}-P G_{u v}\right) \nabla u \nabla v\right](x, t) \\
+\left[\left(2 \delta G_{v}-P G_{v v}\right)|\nabla v|^{2}\right](x, t) \\
\phi(x, t)=\left[G_{u}(u, v) \phi_{1}(u, v)+G_{v}(u, v) \phi_{2}(u, v)\right](x, t) .
\end{gathered}
$$

Then we have

$$
w_{t}(x, t)=P \Delta w+h(x, t)+\phi(x, t), \text { for all }(x, t) \text { satisfying } u(x, t)>K_{0} .
$$

Let

$$
A:=2 \gamma G_{u}-P G_{u u}, \quad B:=\alpha G_{u}-P G_{u v}, \quad C:=2 \delta G_{v}-P G_{v v} .
$$

So, from (2.11) we have

$$
h(x, t)=\left(A|\nabla u|^{2}+2 B \nabla u \nabla v+C|\nabla v|^{2}\right)(x, t), \quad \forall(x, t) \in Q_{T} .
$$


Moreover, on $\Gamma_{K_{0}}$, we have

$$
\begin{aligned}
A & =2 \gamma f G_{v}-\left(f_{u} G_{v}+f G_{u v}\right) P \\
& =\left(2 \gamma f-P f_{u}\right) G_{v}-P f\left(f_{v} G_{v}+f G_{v v}\right) \\
& =\left(2 \gamma f-P f_{u}-P f f_{v}\right) G_{v}-P f^{2} G_{v v}, \\
B & =\alpha f G_{v}-\left(f_{v} G_{v}+f G_{v v}\right) P \\
& =\left(\alpha f-P f_{v}\right) G_{v}-P f G_{v v} .
\end{aligned}
$$

We now define

$$
E(u, v)=\left(2 \gamma f-P f_{u}-P f f_{v}\right)-2\left(\alpha f-P f_{v}\right) f+2 \delta f^{2}
$$

Hence, for $(x, t) \in Q_{T}$ such that $u(x, t)>K_{0}$ and $f(u(x, t), v(x, t)) \nabla u(x, t)+$ $\nabla v(x, t)=0$, we get

$$
h(x, t)=\left[E(u, v) G_{v}|\nabla u|^{2}\right](x, t) .
$$

We have the following lemma.

Lemma 2.3. Assume either $\alpha<2 \delta$ or $\alpha=2 \delta$ and $d_{1} \leq d_{2}$. Then there is $K_{1}>0$ such that for all $0 \leq v \leq m$ and $u \geq K_{1}$, we have $E(u, v) \leq 0$.

Proof. We have

$$
\begin{aligned}
E(u, v) & =\left(2 \gamma f-P f_{u}-P f f_{v}\right)-2\left(\alpha f-P f_{v}\right) f+2 \delta f^{2} \\
& =2 \gamma f+2(\delta-\alpha) f^{2}+P f f_{v}-P f_{u} \\
& =f\left\{2 \gamma+2(\delta-\alpha) f+P f_{v}\right\}-P f_{u} \\
& =f\left\{2 \gamma+2(\delta-\alpha) \frac{d+a u-b v}{u}-\frac{b\left(d_{1}+\alpha v+2 \gamma u\right)}{u}\right\}-P f_{u} \\
& =\frac{f}{u}\left\{[2 \gamma+2(\delta-\alpha) a-2 \gamma b] u-b[2(\delta-\alpha)+\alpha] v-b d_{1}+2(\delta-\alpha) d\right\}-P f_{u} \\
& =\frac{f}{u}\left\{-b(2 \delta-\alpha) v-\frac{2 \delta-\alpha}{\alpha} d_{1}+2(\delta-\alpha) \frac{d_{1}-d_{2}}{\alpha}\right\}-P f_{u} \\
& =\frac{f}{u}\left\{-b(2 \delta-\alpha) v-d_{1}-\frac{2(\delta-\alpha) d_{2}}{\alpha}\right\}-P f_{u} \\
& =\frac{d-b v+a u}{u^{2}}\left\{-b(2 \delta-\alpha) v-d_{1}-\frac{2(\delta-\alpha) d_{2}}{\alpha}\right\} \\
& =\frac{c_{11} u+(d-b v) c_{12}}{u^{2}}, \quad+\frac{(d-b v)\left(d_{1}+\alpha v+2 \gamma u\right)}{u^{2}}
\end{aligned}
$$


where

$$
\begin{aligned}
c_{11} & =a\left\{-b(2 \delta-\alpha) v-d_{1}-\frac{2(\delta-\alpha) d_{2}}{\alpha}\right\}+2 \gamma(d-b v) \\
& =-b\{a(2 \delta-\alpha)+2 \gamma\} v-\frac{2 \gamma}{\alpha}\left[d_{1}+\frac{2(\delta-\alpha) d_{2}}{\alpha}\right]+\frac{2 \gamma\left(d_{1}-d_{2}\right)}{\alpha} \\
& =-\frac{4 b \gamma \delta}{\alpha} v-\frac{2 \gamma}{\alpha^{2}}(2 \delta-\alpha) d_{2}=-\frac{2 b \gamma}{\alpha}\left(d_{2}+2 \delta v\right), \\
c_{12} & =\left\{-b(2 \delta-\alpha) v-d_{1}-\frac{2(\delta-\alpha) d_{2}}{\alpha}\right\}+d_{1}+\alpha v \\
& =-\frac{2(\delta-\alpha) d_{2}}{\alpha}+\left\{\alpha-\frac{(2 \delta-\alpha)^{2}}{\alpha}\right\} v \\
& =\frac{2(\alpha-\delta) d_{2}}{\alpha}+\frac{4 \delta(\alpha-\delta)}{\alpha} v=\frac{2(\alpha-\delta)}{\alpha}\left(d_{2}+2 \delta v\right) .
\end{aligned}
$$

So we have

$$
E(u, v)=\frac{d_{2}+2 \delta v}{\alpha u^{2}}[-2 \gamma b u+2(\alpha-\delta)(d-b v)]
$$

Therefore, from the assumptions of Lemma 2.3, we can choose sufficiently large $K_{1}>0$ such that $E(u, v) \leq 0$ for all $0 \leq v \leq m$ and $u \geq K_{1}$. This completes the proof of Lemma 2.3 .

Now let $K_{2}>\max \left\{K_{0}, K_{1}\right\}$ be sufficiently large so that

$$
\phi_{1}(u, v)<0 \text { and } \phi_{2}(u, v)<0, \quad \forall 0 \leq v \leq m, u \geq K_{2},
$$

where $K_{0}$ is a positive number appearing in (2.5) and $K_{1}$ is a positive number given in Lemma 2.3. Also we define

$$
M_{0}=\max \left\{\sup _{\bar{\Omega}} G\left(u_{0}(x), v_{0}(x)\right), \sup _{(u, v) \in \Gamma \backslash \Gamma_{K_{2}}} G(u, v)\right\}+1 .
$$

Then,

$$
w(x, t) \geq M_{0} \Rightarrow\left(u(x, t, v(x, t)) \in \Gamma_{K_{2}} .\right.
$$

Now, we are ready to apply the maximum principle to the equation (2.13) and get the boundedness of $w$, which in turn implies the boundedness of $u$. We have the following theorem.

Theorem 2.1. Assume either $\alpha<2 \delta$ or $\alpha=2 \delta$ and $d_{1} \leq d_{2}$. Then $w \leq M_{0}$ on $Q_{T}$.

Proof. We introduce

$$
\bar{w}(t):=\sup _{\Omega} w(x, t), \forall t \in[0, T) .
$$

By (2.9) and (2.19), we see that $\bar{w}(0)<M_{0}$. Assume by contradiction that there exists $0<t_{1}<T$ such that $M_{1}=\bar{w}\left(t_{1}\right)>M_{0}$. Let $t_{0}=\inf \left\{t: \bar{w}(t) \geq M_{1}\right\}$. We then see that $0<t_{0} \leq t_{1}, \bar{w}\left(t_{0}\right)=M_{1}$ and $\bar{w}(t)<M_{1}$ for all $0 \leq t<t_{0}$. Let $x_{0}$ be a point in $\bar{\Omega}$ such that $w\left(x_{0}, t_{0}\right)=M_{1}$; then

$$
\frac{\partial w\left(x_{0}, t_{0}\right)}{\partial t} \geq 0
$$


Now, if $x_{0} \in \Omega$, then $\nabla w\left(x_{0}, t_{0}\right)=0$. Moreover, if $x_{0} \in \partial \Omega$, then

$$
\frac{\partial w\left(x_{0}, t_{0}\right)}{\partial \nu}=G_{u} \frac{\partial u\left(x_{0}, t_{0}\right)}{\partial \nu}+G_{v} \frac{\partial v\left(x_{0}, t_{0}\right)}{\partial \nu}=0
$$

and the tangential derivative $\nabla_{\partial \Omega} w\left(x_{0}, t_{0}\right)=0$. So $\nabla w\left(x_{0}, t_{0}\right)=0$ for both cases. By (2.20), we have $\left(u\left(x_{0}, t_{0}\right), v\left(x_{0}, t_{0}\right)\right) \in \Gamma_{K_{2}}$. Therefore, by Lemma 2.2 we have

$$
f\left(u\left(x_{0}, t_{0}\right), v\left(x_{0}, t_{0}\right)\right) \nabla u\left(x_{0}, t_{0}\right)+\nabla v\left(x_{0}, t_{0}\right)=\frac{1}{G_{v}} \nabla w\left(x_{0}, t_{0}\right)=0 .
$$

So by Lemma 2.2, Lemma 2.3, (2.16), (2.18) and (2.23), we see that

$$
\begin{aligned}
& h\left(x_{0}, t_{0}\right)=\left(E(u, v) G_{v}|\nabla u|^{2}\right)\left(x_{0}, t_{0}\right) \leq 0, \\
& \phi\left(x_{0}, t_{0}\right)<0,
\end{aligned}
$$

where $h, \phi$ and $E$ are defined by (2.11), (2.12) and (2.15), respectively. Now if $x_{0} \in \Omega$, we have $\Delta w\left(x_{0}, t_{0}\right) \leq 0$. From (2.13) we have

$$
w_{t}\left(x_{0}, t_{0}\right)<0 \text {. }
$$

This contradicts (2.22). On the other hand, if $x_{0} \in \partial \Omega$, we see from (2.13), (2.24) and (2.25) that in a neighborhood of $\left(x_{0}, t_{0}\right)$,

$$
w_{t}-P \Delta w \leq 0 \text {. }
$$

Then, by Hopf's boundary point lemma (see 14]) we also get a contradiction. This completes the proof of Theorem 2.1

\section{Proof of Theorem 1.2}

By (2.9), Lemma 2.2 and Theorem 2.1 we see that $u$ is uniformly bounded. Therefore, the proof of Theorem 1.2 is now exactly the same as that of Theorem 1.1 of [6]. We give it here for completeness. First, we write

$$
v_{t}=\nabla \cdot\left[\left(d_{2}+2 \delta v\right) \nabla v\right]+v\left(a_{2}-b_{2} u-c_{2} v\right) .
$$

Since $d_{2}+2 \delta v$ and $v\left(a_{2}-b_{2} u-c_{2} v\right)$ are bounded in $\bar{Q}_{T}$, applying Theorem 1.3 in [7, p.43] to (3.11) (see also Theorem 10.1 in [9, p.204]), we see that there is a constant $\beta>0$ such that

$$
v \in C^{\beta, \beta / 2}\left(\bar{Q}_{T}\right) .
$$

Let $w_{1}=\left(d_{2}+\delta v\right) v$. Then we have

$$
\frac{\partial w_{1}}{\partial t}=\left(d_{2}+2 \delta v\right) \Delta w_{1}+f_{1},
$$

where $f_{1}=\left(d_{2}+2 \delta v\right) \phi_{2}(u, v)$. Since $d_{2}+2 \delta v$ is in $C^{\beta, \beta / 2}\left(\bar{Q}_{T}\right)$ and $f_{1}$ is bounded in $\overline{Q_{T}}$, by Theorem 9.1 [9, pp. 341-342] and its remark [9, p.351], we have

$$
\left\|w_{1}\right\|_{W_{q}^{2,1}\left(Q_{T}\right)}<\infty, \forall q>1 \text {. }
$$

Choosing a sufficiently large $q$ and using Lemma 3.3 in [9, p.80], we get

$$
w_{1} \in C^{1+\beta^{*},\left(1+\beta^{*}\right) / 2}\left(\overline{Q_{T}}\right) \text { for any } 0<\beta^{*}<1 .
$$

Since $v=\left\{-d_{2}+\sqrt{\left.d_{2}^{2}+4 \delta w_{1}\right)}\right\} /(2 \delta)$, it follows from (3.3) that

$$
v \in C^{1+\beta^{*},\left(1+\beta^{*}\right) / 2}\left(\overline{Q_{T}}\right) \text { for any } 0<\beta^{*}<1 .
$$

Now, $u$ is a solution of

$$
u_{t}=\nabla \cdot\left[\left(d_{1}+\alpha v+2 \delta u\right) \nabla u+\alpha u \nabla v\right]+f_{2},
$$


where $u, v, \nabla v$ and $f_{2}=\phi_{1}(u, v)$ are all bounded. By [7, Theorem 1.3, p.43], we have

$$
u \in C^{\sigma, \sigma / 2}\left(\overline{Q_{T}}\right) \text { with some } 0<\sigma<1 .
$$

Now the equation of $v$ can be written as

$$
v_{t}=\left(d_{2}+2 \delta v\right) \Delta v+f_{3},
$$

where $f_{3}=2 \delta|\nabla v|^{2}+\phi_{2}(u, v) \in C^{\sigma, \sigma / 2}\left(\overline{Q_{T}}\right)$ by (3.4) and (3.5). Then the Schauder estimate (see Theorem 5.3 in [9. pp.320-321]) applied to equation (3.6) yields

$$
v \in C^{2+\sigma^{*},\left(2+\sigma^{*}\right) / 2}\left(\overline{Q_{T}}\right) \text { with } \sigma^{*}=\min \{\lambda, \sigma\} .
$$

Now let $w_{2}=\left(d_{1}+\alpha v+\gamma u\right) u$. Then $w_{2}$ satisfies

$$
\frac{\partial w_{2}}{\partial t}=\left(d_{1}+\alpha v+2 \delta u\right) \Delta w_{2}+f_{4},
$$

where $f_{4}=\left(d_{1}+\alpha v+2 \delta u\right) \phi_{1}(u, v)+\alpha u v_{t} \in C^{\sigma^{*}, \sigma^{*} / 2}\left(\overline{Q_{T}}\right)$ by (3.5) and (3.7). From this, $d_{1}+\alpha v+2 \delta u \in C^{\sigma, \sigma / 2}\left(\overline{Q_{T}}\right)$ (by $(3.5)$ ) and by applying the Schauder estimate to the equation (3.8), we have

$$
w_{2} \in C^{2+\sigma^{*},\left(2+\sigma^{*}\right) / 2}\left(\overline{Q_{T}}\right) .
$$

Then

$$
u=\left\{-\left(d_{1}+\alpha v\right)+\sqrt{\left(d_{1}+\alpha v\right)^{2}+4 \gamma w_{2}}\right\} /(2 \gamma) \in C^{2+\sigma^{*},\left(2+\sigma^{*}\right) / 2}\left(\overline{Q_{T}}\right) .
$$

Now repeating the procedure by making use of (3.7) and (3.10) in place of (3.4) and (3.5), we have

$$
u, v \in C^{2+\lambda,(2+\lambda) / 2}\left(\overline{Q_{T}}\right) .
$$

Finally, the estimates (3.7) and (3.10) imply that the hypotheses of Theorem 1.1 are satisfied. Therefore, the solution $(u, v)$ exists globally in time. The proof of Theorem 1.2 is now complete.

Remark 3.1. Theorem 1.2 still holds with general $\phi_{1}, \phi_{2}$ if we assume that $\phi_{1}, \phi_{2}$ are continuous, negative when $u$ or $v$ is sufficiently large and $\phi_{1}(0,)=.\phi_{2}(., 0)=0$. Our results also hold for the Dirichlet boundary condition.

\section{ACKNOWLEDGMENTS}

I would like to thank Professors Wei-Ming Ni and Peter Poláčik for their comments, encouragement and useful discussion. I also would like to thank the anonymous referees for a very careful reading of the manuscript and helpful suggestions.

\section{REFERENCES}

[1] H. Amann, Dynamic theory of quasilinear parabolic equations - I. Abstract evolution equations, Nonlinear Analysis - Theory, Methods and Applications, Vol. 12, 9(1988), 895-919. MR960634 (89j:35072)

[2] H. Amann, Dynamic theory of quasilinear parabolic equations - II. Reaction-diffusion systems, Differential Integral Equations, Vol. 3, 1(1990), 13-75. MR.1014726 (90i:35124)

[3] H. Amann, Dynamic theory of quasilinear parabolic systems - III. Global existence, Math. Z. 202(1989), 219-250. MR1013086 (90i:35125)

[4] H. Amann, Abstract methods in differential equations, Rev. R. Acad. Cien. Serie A. Mat. Vol. 97, 1(2003), 89-105. MR2037227 (2004k:35231) 
[5] Y.S. Choi, R. Lui and Y. Yamada, Existence of global solutions for the Shigesada-KawasakiTeramoto model with weak cross-diffusion, Discrete and Continuous Dynamical Systems, Vol. 9, 5(2003), 1193-1200. MR.1974423 (2004b:35145)

[6] Y.S. Choi, R. Lui and Y. Yamada, Existence of global solutions for the Shigesada-KawasakiTeramoto model with strongly coupled cross-diffusion, Discrete and Continuous Dynamical Systems, Vol. 10, 3(2004), 719-730. MR2018876 (2005e:35099)

[7] E. DiBenedetto, Degenerate Parabolic Equations, Springer-Verlag, New York, 1993. MR.1230384 (94h:35130)

[8] A. Friedman, Partial Differential Equations of Parabolic Type, Prentice-Hall, Englewood Cliffs, N.J., 1964. MR0181836 (31:6062)

[9] O.A. Ladyzenskaja, V.A. Solonnikov and N.N. Ural'ceva, Linear and Quasi-linear Equations of Parabolic Type, Translations of Mathematical Monographs, 23, AMS, 1968. MR0241821 $(39: 3159 a)$

[10] G. Lieberman, Second Order Parabolic Differential Equations, World Scientific, 1996. MR:1465184 (98k:35003)

[11] D. Le and T. Nguyen, Global existence for a class of triangular parabolic systems on domains of arbitrary dimension, Proceedings of AMS, Vol. 133, 7(2005), 1985-1992. MR2137864 (2005k:35215)

[12] Y. Li and C. Zhao, Global existence of solutions to a cross-diffusion system in higher dimensional domains, Discrete and Continuous Dynamical Systems, Vol. 12, 2(2005), 185-192. MR2122161 (2005i:35147)

[13] Y. Lou, W.M. Ni and Y. Wu, On the global existence of cross-diffusion systems, Discrete and Continuous Dynamical Systems, Vol. 4, 2(1998), 193-203. MR1616969 (99f:35089)

[14] M.H. Protter and H.F. Weinberger, Maximum Principles in Differential Equations, Second Edition, Springer-Verlag, New York, 1984. MR762825 (86f:35034)

[15] N. Shigesada, K. Kawasaki and E. Teramoto, Spatial segregation of interacting species, J. Theoretical Biology, 79(1979), 83-89. MR540951 (80e:92038)

[16] J. Smoller, Shock Waves and Reaction-Diffusion Equations. Springer, New York (1983).

School of Mathematics, University of Minnesota, 206 Church Street S.E., MinneapoLis, Minnesota 55455

E-mail address: phan@math.umn.edu 